\section{Cureus}

Received 02/26/2019

Review began 03/01/2019

Review ended 03/02/2019

Published 03/06/2019

\section{(c) Copyright 2019}

Prabhat et al. This is an open access article distributed under the terms of the Creative Commons Attribution License CC-BY 3.0., which permits unrestricted use, distribution, and reproduction in any medium, provided the original author and source are credited.

\title{
Spinal Epidural Abscess in Melioidosis: A Rare Case Report from Eastern India
}

\author{
Vinay Prabhat ${ }^{1}$, Amrit Gantaguru ${ }^{2}$, Sudarsan Behera ${ }^{1}$, Rajesh Rana ${ }^{1}$, Gurudip Das ${ }^{1}$ \\ 1. Orthopaedics, All India Institute of Medical Sciences, Bhubaneswar, IND 2. Orthopedics, All India \\ Institute of Medical Sciences, Bhubaneswar, IND
}

$\square$ Corresponding author: Amrit Gantaguru, amrit.sunny87@gmail.com Disclosures can be found in Additional Information at the end of the article

\section{Abstract}

A spinal epidural abscess (SEA) is a rare condition that has a devastating impact on the patient's health. It is difficult to diagnose and can present with a myriad of symptoms with or without the involvement of a neurological deficit. The conditions that lead to immunocompromised status, such as malnutrition, diabetes, intravenous drug abuse, previous surgical intervention, and human immunodeficiency virus (HIV) infection/acquired immune deficiency syndrome (AIDS) can predispose a patient to SEA. The most common organisms isolated from the affected patient include Staphylococcus aureus and Streptococcus species while an abscess in some cases can be caused by tuberculosis and fungal and parasitic infections. Among the other causative organisms is Burkholderia pseudomallei (B. pseudomallei), also known as Pseudomonas pseudomallei, which is a Gram-negative, bipolar, aerobic, motile, and rod-shaped bacterium. It is a soil-dwelling bacterium, which is endemic in tropical and subtropical regions worldwide, particularly in Thailand and northern Australia, and causes melioidosis. To our knowledge, SEA caused by B. pseudomallei from the Indian subcontinent has not been reported in the literature. In this case report, we present the case of a patient with SEA caused by B. pseudomallei.

Categories: Infectious Disease, Neurosurgery, Orthopedics

Keywords: spinal epidural abscess, melioidosis, paraparesis

\section{Introduction}

Spinal epidural abscess (SEA) is a rare but potentially devastating condition, which may lead to a permanent neurological deficit and even death if not managed promptly. It is caused by an infection of the soft tissues and bone of the spinal column characterized by the accumulation of pus in the epidural space, which leads to spinal cord compression. The incidence of SEA has been increasing in the last two-three decades, from $0.2-1$ case to $2.5-3$ cases per 10,000 hospital admissions [1]. Usually, SEA patients present with a complaint of mild back pain and fever before the onset of acute neurological deterioration [2-4]. However, the classical triad of fever, back pain, and neurologic deficit is found in only $10 \%-15 \%$ of patients with SEA who present to the physician for the first time [2-3]. If the diagnosis is delayed, the further evolution of symptoms occurs in four phases: (1) localized spinal pain; (2) radicular pain and paresthesia; (3) muscular weakness, sensory loss, and sphincter dysfunction; and (4) paralysis [4]. Only a few cases of SEA with paraplegia have been reported till date [5]. SEA with paraplegia is an emergency condition that requires the urgent decompression of the spinal cord, as it may lead to a permanent neurological deficit if left untreated. The pathophysiology involved in the neurological deterioration in SEA mainly includes mechanical obstruction and pressure necrosis [6]. 
The most common causative organism of SEA is Staphylococcus aureus while other organisms, such as Escherichia coli, coagulase-negative staphylococci, Bacteroides species and anaerobes, Pseudomonas species and Streptococci, including Streptococcus viridans, group B streptococci, and pneumococcus also cause this condition [1,7-8]. To our knowledge, SEA caused by Burkholderia pseudomallei from the eastern region of the Indian subcontinent has not been reported. B. pseudomallei is a Gram-negative bacillus, which is endemic in the tropical and subtropical regions but is usually not endemic to India. It is the causative agent of melioidosis and causes abscesses in the lung, liver, spleen, parotid glands, and skeletal muscles [9-11]. Patients with immunocompromised status, such as those with diabetes mellitus, chronic renal failure, and alcoholic cirrhosis, are more susceptible to SEA [12-13]. In this case report, we present a rare case of SEA with paraparesis caused by Burkholderia in a patient with type II diabetes. The patient initially presented with low back pain mimicking sacroiliitis, but later, the condition was diagnosed as SEA and managed with midline posterior decompression and evacuation of pus along with antibiotics.

\section{Case Presentation}

A 28-year-old male patient presented in casualty with complaints of fever with chills and rigors with pain in the abdomen for a period of 20 days. The patient had initially taken antipyretics and antibiotics but found no effect. On clinical examination, the patient was febrile, and his abdomen was soft without any guarding or rigidity. Routine investigation showed that the patient had diabetes, which was uncontrolled. The test results indicated that the patient also had a high erythrocyte sedimentation rate along with a high total leukocyte count and a high level of positive C-reactive protein. Therefore, insulin and empirical antibiotics were started immediately. An ultrasonographic examination of the abdomen revealed hepatomegaly, chronic pancreatitis, and splenomegaly with multiple splenic abscesses. An endoscopic examination of the upper gastrointestinal tract showed the presence of esophageal candidiasis with a dilated vein at the fundus. Contrast-enhanced computed tomography (CECT) of the abdomen indicated chronic calcific pancreatitis with splenic vein thrombosis, multiple splenic abscesses with evidence of rupture and ascites, and bilateral pleural effusion with basal lung collapse. Blood culture report showed the presence of B. pseudomallei, which was sensitive to imipenem and aminoglycosides, and so the patient was put on antibiotics. A few days through the course of treatment, the patient complained of low back pain. Clinical examination revealed tenderness at the bilateral sacroiliac joints with no neurological deficit. Plain radiographic examination confirmed the diagnosis of bilateral sacroiliitis and, therefore, twicedaily sulfasalazine $500 \mathrm{mg}$ was added to the treatment regimen. One week later, the patient presented again with unimproved back pain and paraesthesia and weakness in both the lower limbs. On musculoskeletal examination, the power of both the lower limbs was found to be $4 / 5$, with decreased sensation in the bilateral $\mathrm{L}_{4}, \mathrm{~L}_{5}$, and $\mathrm{S}_{1}$ dermatomes. An examination of the upper limb showed no neurological deficit. Magnetic resonance imaging (MRI) of the spine showed abnormal hyper-intensity of $\mathrm{C}_{5}, \mathrm{D}_{1}, \mathrm{D}_{8}$, and $\mathrm{D}_{12}$ vertebra body with post-contrast nonenhancement of the portion of $\mathrm{D}_{12}$ vertebra along with anterior epidural, left paraspinal, left iliac, and sacroiliac abscesses (Figures $1 A-1 C$ and Figures $2 A-2 B$ ). 


\section{Cureus}
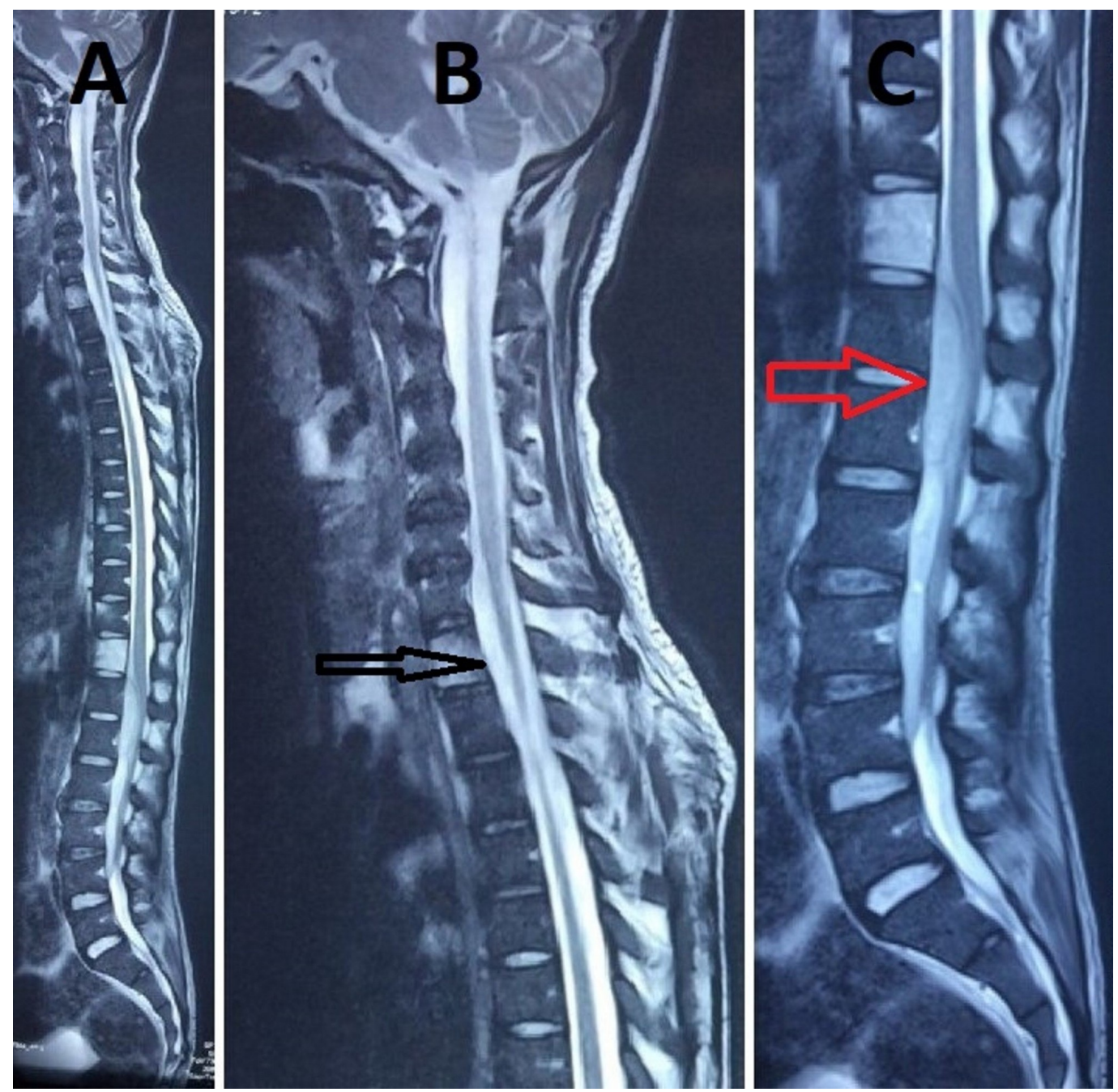

FIGURE 1: Magnetic resonance imaging (MRI) of whole spine (sagittal section)

$1 \mathrm{~A}: \mathrm{MRI}$ of whole showing lesion in the cervical and lumbar region

1B: MRI of the cervical spine showing epidural abscess $\left(\mathrm{C}_{5}-\mathrm{T}_{5}\right)$ (arrow mark)

1C: MRI of the dorsolumbar spine showing epidural abscess $\left(D_{12}-L_{4}\right)$ (arrow mark)

MRI: magnetic resonance imaging 


\section{Cureus}

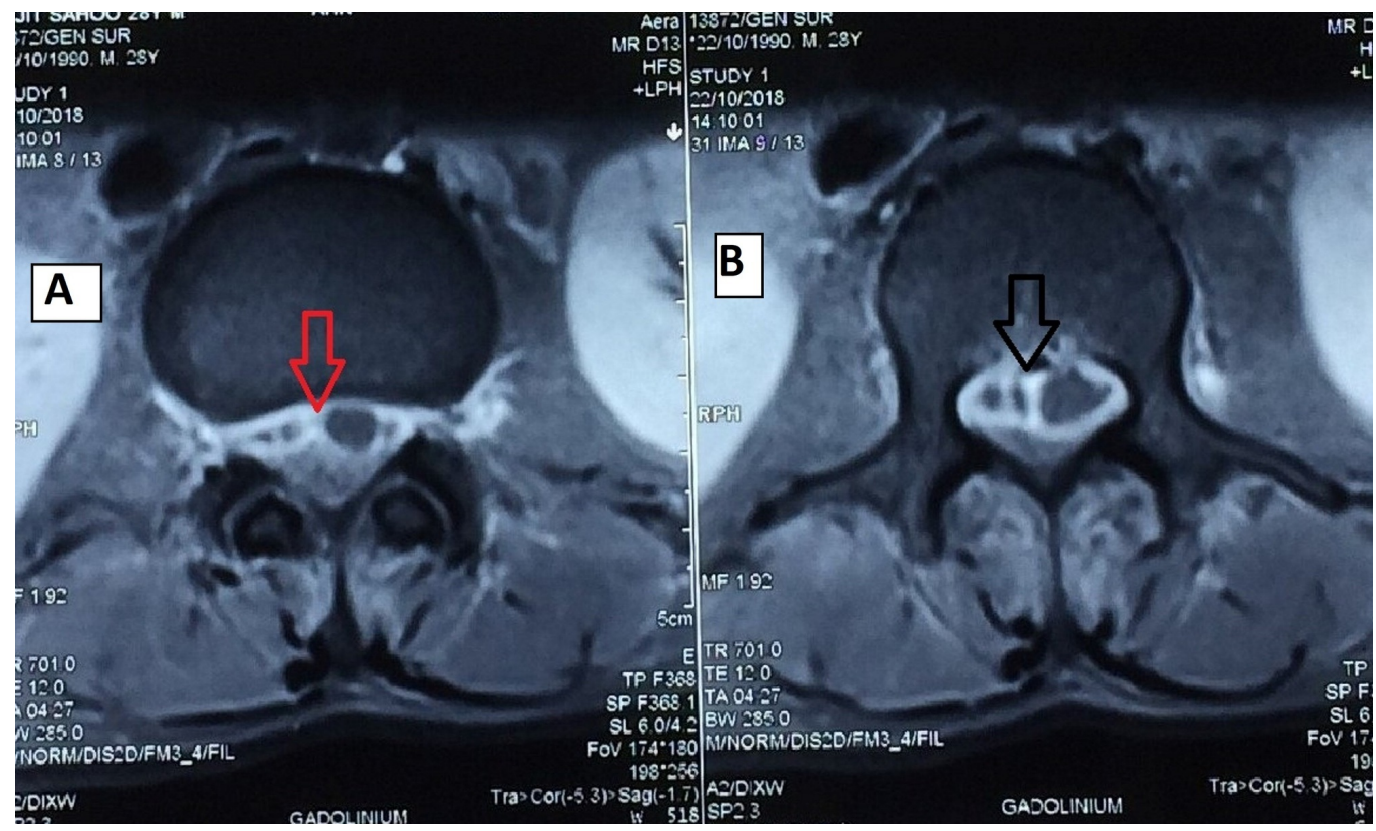

FIGURE 2: A and B: MRI (axial section) showing a lobulated abscess in the epidural space (arrow mark)

MRI: magnetic resonance imaging

Bilateral sacroiliitis was also confirmed by MRI examination, so emergency decompression was planned for the patient. Accordingly, a laminectomy was performed, extending from $D_{12}$ to $L_{4}$ vertebra without affecting facet joints and the intraoperative abscess was found to be loculated (Figures 3A-3C).

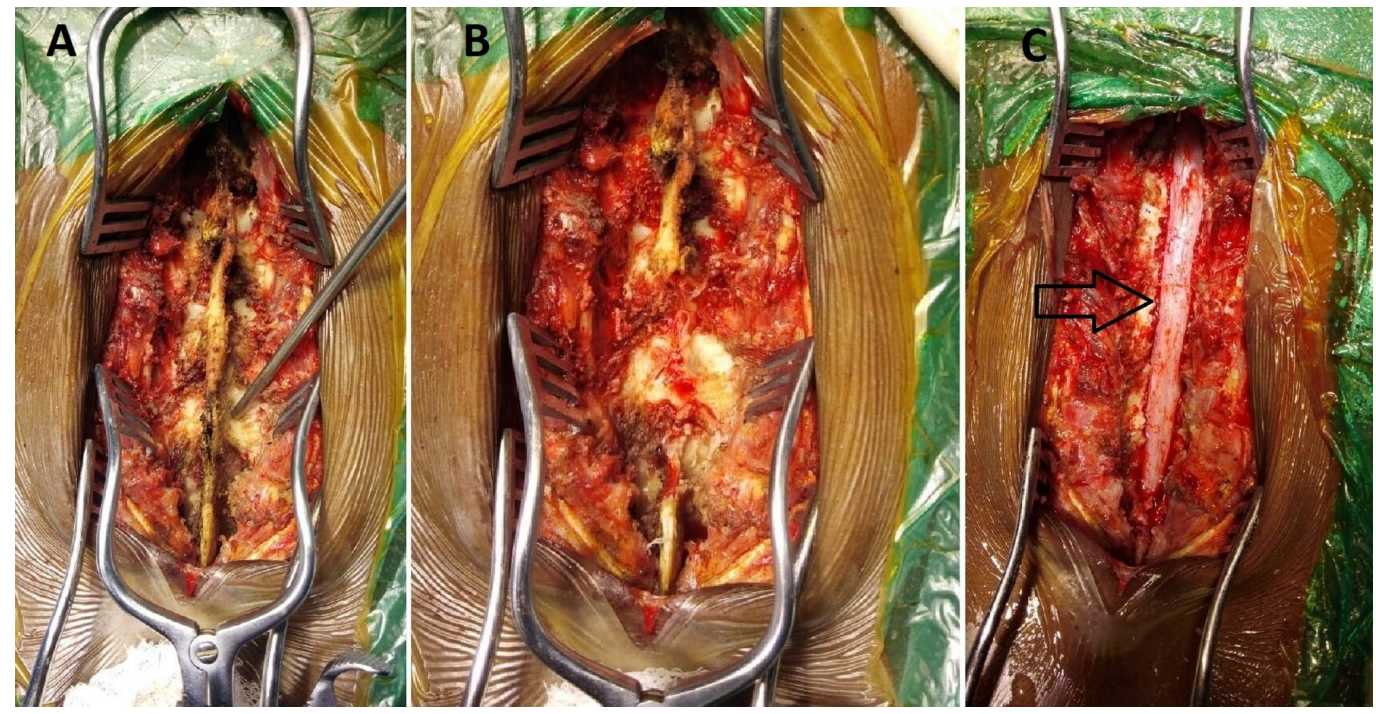

FIGURE 3: Intraoperative clinical photographs

3A: A clinical photograph showing D11-L5

3B: Removal of the spinous process from D12 to L4

3C: A clinical image of the spinal cord (arrow) after decompression 
Proper decompression with an evacuation of pus was done, and the obtained pus was sent to the laboratory for culture and sensitivity analysis. The culture and sensitivity report was suggestive of the growth of B. pseudomallei, which was sensitive to imipenem and aminoglycosides. Postoperatively, the neurological status of the patient improved, and he was shifted to the general surgery unit where he was operated for a splenic abscess. Intravenous antibiotics were continued for six weeks, and the patient was discharged. At the three-month follow-up, the patient had recovered completely.

\section{Discussion}

SEA usually presents as fever and back pain, and if the diagnosis is delayed, the symptoms evolve into paraplegia [2-4]. The patient who presented to us had fever, back pain, and abdominal pain initially, and after 25 days of initial presentation, he developed symptoms of neurological deficit. The diagnosis of SEA was delayed, as its incidence is very rare and the classical triad of fever, back pain, and neurological deficit occurs in only $10 \%-15 \%$ of all those who present with SEA for the first time [1-3]. MRI is usually performed to best visualize the location and extent of abscesses, including those involving spinal, paraspinal, and disc regions, as compared to other imaging techniques, such as CT myelography [14]. In our case, the MRI examination revealed a hyperintensity of the $C_{5}, D_{1}, D_{8}$, and $D_{12}$ vertebra body with an anterior epidural abscess from $\mathrm{D}_{12}$ to $\mathrm{L}_{4}$ and $\mathrm{C}_{5}$ to $\mathrm{D}_{1}$, along with left paraspinal, left iliac, and sacroiliac paraspinal abscesses. The clinical symptoms of the patient suggested paraplegia due to abscess from $\mathrm{D}_{12}$ to $\mathrm{L}_{4}$, but no neurological signs suggestive of abscess in the cervical region were seen. As early intervention gives better results, surgical decompression was planned for the abscess in the dorsolumbar region at the earliest [15-16]. As soon as the patient was admitted, empirical antibiotics were started based on the blood culture and sensitivity report. Decompression and evacuation of pus were done following laminectomy from $\mathrm{D}_{12}$ to $\mathrm{L}_{4}$, as the abscess was found to be loculated and thus could not be drained at a single level, but the facet joint was preserved to maintain stability (Figure 3) [17]. Unlike a single-level spinal dural abscess, which could be managed by percutaneous CT-guided aspiration with antibiotics, a multiple-level abscess, which is lobulated, needs open decompression [18]. The patient was treated with parenteral antibiotics for six weeks followed by treatment with oral antibiotics for six weeks based on the sensitivity report.

The most common causative organism of SEA is S. aureus [1,7-8], but in our case, the causative organism was found to be B. pseudomallei, which was detected preoperatively in blood culture and in pus, which was obtained during drainage and cultured for sensitivity analysis. B. pseudomallei is a Gram-negative bacillus that causes abscesses in the liver, lungs, spleen, parotid glands, and skeletal muscles in immunocompromised patients, including those with diabetes, chronic kidney disease, and alcoholic cirrhosis living in the endemic areas in East Asia, especially Thailand and northern Australia [9-13]. Although India is not an endemic area, our patient had uncontrolled diabetes when he presented to us with an abscess in the spleen, liver, and spinal epidural space. Probably, this is the first case report of SEA with paraparesis caused by B. pseudomallei.

\section{Conclusions}

SEA is an uncommon condition and should be considered a differential diagnosis in patients presenting with fever and back pain so that it can be diagnosed early and treated promptly to prevent a permanent neurological deficit. Our case showed that though India is not an endemic country for B. pseudomallei, this organism may cause SEA in immunocompromised patients. 


\section{Additional Information \\ Disclosures}

Human subjects: Consent was obtained by all participants in this study. Conflicts of interest: In compliance with the ICMJE uniform disclosure form, all authors declare the following:

Payment/services info: All authors have declared that no financial support was received from any organization for the submitted work. Financial relationships: All authors have declared that they have no financial relationships at present or within the previous three years with any organizations that might have an interest in the submitted work. Other relationships: All authors have declared that there are no other relationships or activities that could appear to have influenced the submitted work.

\section{References}

1. Sendi P, Bregenzer T, Zimmerli W: Spinal epidural abscess in clinical practice . QJM-Int J Med. 2008, 101:1-12. 10.1093/qjmed/hcm100

2. Joshi SM, Hatfield RH, Martin J: Spinal epidural abscess: a diagnostic challenge . Br J Neurosurgery. 2003, 17:160-163.

3. Davis DP, Wold RM, Patel RJ, Tran AJ, Tokhi RN, Chan TC, Vilke GM: The clinical presentation and impact of diagnostic delays on emergency department patients with spinal epidural abscess. J Emerg Med. 2004, 26:285-291. 10.1016/j.jemermed.2003.11.013

4. Darouiche RO: Spinal epidural abscess. N Engl J Med. 2006, 355:2012-2020. 10.1056/NEJMra055111

5. Stephanides GC, Gibson RM: Paraplegia caused by spinal epidural abscess . Postgrad Med J. 1984, 64:603-605. 10.1136/pgmj.64.754.603

6. Feldenzer JA, McKeever PE, Schaberg DR: Experimental spinal epidural abscess: a pathophysiological model in the rabbit. Neurosurgery. 1987, 20:859-867.

7. Reihsaus E, Waldbaur H, Seeling W: Spinal epidural abscess: a meta-analysis of 915 patients . Neurosurgery. 2000, 23:175-204.

8. Gerberding JL, Romero JM, Ferraro MJ: Case records of the Massachusetts General Hospital. Case 34-2008. A 58-year-old woman with neck pain and fever. N Engl J Med. 2008, 359:19421949. 10.1056/NEJMcpc0806980

9. Rode JW, Webling DD: Melioidosis in the northern territory of Australia . Med J Aust. 1981, $1: 181-184$.

10. Thin RN, Brown M, Stewart JB: Melioidosis: a report of ten cases . Q J Med. 1970, 39:115-127.

11. Pandey V, Rao SP, Rao S: Burkholderia pseudomallei musculoskeletal infections (melioidosis) in India. Indian J Orthopaedics. 2010, 44:216-220.

12. Chaowagul W, White NJ, Dance DA, et al.: Melioidosis: a major cause of community-acquired septicemia in northeastern Thailand. J Infect Disease. 1989, 159:890-899.

13. Curric BJ, Fisher DA, Howard DM, et al.: Endemic melioidosis in tropical northern Australia: a 10-year prospective study and review of the literature. Clin Infect Disease. 2000, 31:981-986.

14. Smith AS, Blaser SI: MR of infectious and inflammatory diseases of the spine . Crit Rev Diagn Imaging. 1991, 32:165-189.

15. Pereira CE, Lynch JC: Spinal epidural abscess: an analysis of 24 cases . Surg Neurol. 2005, 63:26-29. 10.1016/j.surneu.2004.09.021

16. Patel AR, Alton TB, Bransford RJ, Lee MJ, Bellabarba CB, Chapman JR: Spinal epidural abscesses: risk factors, medical versus surgical management, a retrospective review of 128 cases. Spine J. 2014, 14:326-330. 10.1016/j.spinee.2013.10.046

17. Sharma M1, Langrana NA, Rodriguez J: Role of ligaments and facets in lumbar spinal stability . Spine (Phila Pa 1976). 1995, 20:887-900.

18. Lyu RK, Chen CJ, Tang LM: Spinal epidural abscess successfully treated with percutaneous, computed tomography-guided, needle aspiration and parenteral antibiotic therapy: case report and review of the literature. Neurosurgery. 2002, 51:509-512. 